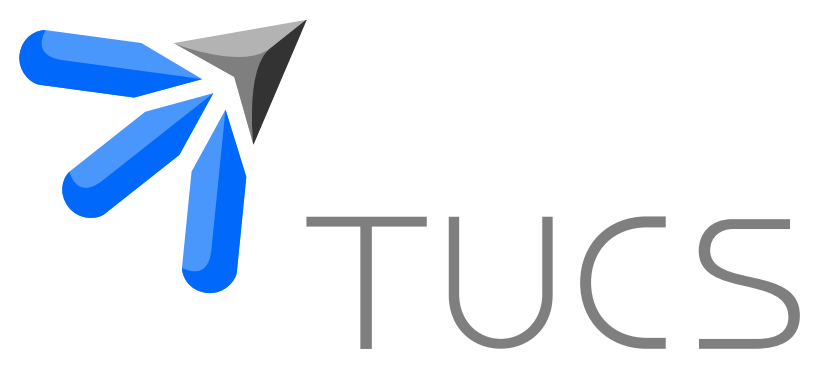

Andrzej Ehrenfeucht | Tero Harju | Grzegorz Rozenberg

\title{
Embedding Linear Orders in Grids
}

TUCS Technical Report

No 636, November 2004 



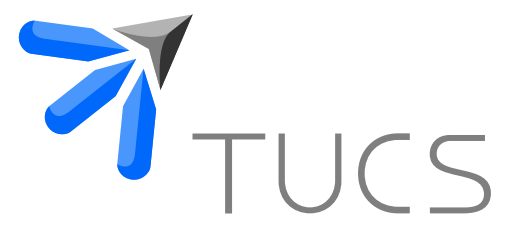

\title{
Embedding Linear Orders in Grids
}

\author{
Andrzej Ehrenfeucht \\ Department of Computer Science \\ University of Colorado at Boulder, Boulder \\ CO 80309-0347, USA \\ email andrzej@cs.colorado.edu \\ Tero Harju \\ Department of Mathematics \\ University of Turku \\ FIN-20014 Turku, Finland, \\ email harju@utu.fi \\ Grzegorz Rozenberg \\ Leiden Institute for Advanced Computer Science \\ Leiden University \\ 2333 CA Leiden, the Netherlands \\ email rozenber@liacs.nl
}

TUCS Technical Report

No 636, November 2004 


\begin{abstract}
A grid is a two-dimensional permutation: an $m \times n$-grid of size $m n$ is an $m \times n$-matrix where the entries run through the elements $\{1,2, \ldots, m n\}$. We prove that if $\delta_{1}$ and $\delta_{2}$ are any two linear orders on $\{1,2, \ldots, N\}$, then they can be simultaneously embedded (in a well defined sense) into a unique grid having the smallest size.
\end{abstract}

Keywords: Linear order, biorder, matrix, grid, embedding

\author{
TUCS Laboratory
}

Discrete Mathematics for Information Technology 


\section{Introduction}

Let $D$ be a finite set of cardinality $n$. A linear order (or a permutation) on $D$ is a sequence $\delta=\left(x_{1}, x_{2}, \ldots, x_{n}\right)$ such that each element $x \in D$ occurs exactly once in $\delta$. Also, if $\delta_{1}$ and $\delta_{2}$ are two linear orders on a common set $D=\{1,2, \ldots, N\}$ for some $N \geq 1$, then the pair $\tau=\left(\delta_{1}, \delta_{2}\right)$ is called a biorder (on $D$ ).

We shall study the problem of representing biorders in the form of grids, i.e., matrices that have all entries different from each other. We show that each biorder $\tau$ has a unique (in a well defined sense) grid of the smallest size representing it.

A biorder $\left(\delta_{1}, \delta_{2}\right)$ can be regarded as a partial order $\rho=\delta_{1} \cap \delta_{2}$ of dimension two; see Trotter [5] for the results on the dimensions of partially ordered sets. A theorem of Dushnik and Miller states that a partially ordered set $P$ has dimension at most two if and only if the incomparability graph of $P$ is also a comparability graph. It is shown by Pnueli, Lempel and Even [4] that the partially ordered sets of dimension two are closely related to permutation graphs. Indeed, for a permutation graph $G=(D, E)$ one can find a biorder $\left(\delta_{1}, \delta_{2}\right)$ on $D$ such that $a b \in E$ if and only if $(a, b) \in \delta_{1} \cap \delta_{2}$.

Another graph theoretical application of biorders is given in [3], see also $[1,2]$, where the notion of a text is introduced as an ordered triple $\tau=$ $\left(\lambda, \delta_{1}, \delta_{2}\right)$ consisting of a function $\lambda: D \rightarrow S$ from the finite domain $D$ into another set $S$ (say, a word semigroup $A^{*}$ ) and of a biorder $\left(\delta_{1}, \delta_{2}\right)$ on $D$. In a sense a text is a structured word.

\section{Preliminaries}

We denote by $[n, m]$ the interval $\{n, n+1, \ldots, m\}$ of integers. For pairs of integers, $(m, n)<(p, q)$ means that $m \leq p, n \leq q$ and $(m, n) \neq(p, q)$.

We shall often identify a singleton set $\{x\}$ with its element $x$.

Let $\rho$ be a partial order on a finite set $D$, called the domain of $\rho$ and denoted by $\operatorname{dom}(\rho)$. All domains in this paper will be finite, and without loss of generality we shall consider domains consisting of positive integers. The dual order of $\rho$ is the partial order $\rho^{-1}=\{(x, y) \mid(y, x) \in \rho\}$.

The structure preserving functions, i.e., embeddings, considered in this paper preserve partial orders. To be more precise, let $\rho_{1}$ and $\rho_{2}$ be partial orders on the domains $D_{1}$ and $D_{2}$, respectively. A mapping $\varphi: D_{1} \rightarrow D_{2}$ is order preserving, if $\varphi\left(\rho_{1}\right) \subseteq \rho_{2}$, where $\varphi$ maps the relation $\rho_{1}$ pointwise, i.e.,

$$
\varphi\left(\rho_{1}\right)=\left\{(\varphi(x), \varphi(y)) \mid(x, y) \in \rho_{1}\right\} .
$$

An injective order preserving function $\varphi$ is an order embedding.

Let $\rho_{1}$ and $\rho_{2}$ be disjoint partial orders, i.e., $\operatorname{dom}\left(\rho_{1}\right) \cap \operatorname{dom}\left(\rho_{2}\right)=\emptyset$. Then their (directed) sum is the partial order

$$
\rho_{1} \oplus \rho_{2}=\rho_{1} \cup \rho_{2} \cup\left\{(x, y) \mid x \in D_{1} \text { and } y \in D_{2}\right\} .
$$


Also, we adopt the convention that $\rho \oplus \emptyset=\rho=\emptyset \oplus \rho$. Clearly, the operation $\oplus$ is associative on disjoint partial orders, and therefore we can write

$$
\sum_{i=1}^{n} \rho_{i}=\rho_{1} \oplus \rho_{2} \oplus \cdots \oplus \rho_{n}
$$

for the unique sum $\rho_{1} \oplus\left(\rho_{2} \oplus\left(\cdots \oplus\left(\rho_{n-1} \oplus \rho_{n}\right)\right)\right)$ of pairwise disjoint partial orders $\rho_{i}, i=1,2, \ldots, n$. Note, however, that the operation $\oplus$ is not commutative.

\section{Grid biorders}

Let $\delta_{1}$ and $\delta_{2}$ be linear orders on a common domain $[1, N]$ for some $N \geq 1$. Recall that the pair $\tau=\left(\delta_{1}, \delta_{2}\right)$ is a biorder. The domain of $\tau$ is the common domain of its components, $\operatorname{dom}(\tau)=[1, N]$.

If $\tau=\left(\delta_{1}, \delta_{2}\right)$ is a biorder, and $\delta_{1}^{\prime}$ and $\delta_{2}^{\prime}$ are two linear orders with $\operatorname{dom}\left(\delta_{1}^{\prime}\right)=\operatorname{dom}\left(\delta_{2}^{\prime}\right)$ such that $\delta_{1}^{\prime} \subseteq \delta_{1}$ and $\delta_{2}^{\prime} \subseteq \delta_{2}$, then we write $\left(\delta_{1}^{\prime}, \delta_{2}^{\prime}\right) \subseteq \tau$.

Let $\tau=\left(\delta_{1}, \delta_{2}\right)$ and $\tau^{\prime}=\left(\delta_{1}^{\prime}, \delta_{2}^{\prime}\right)$ be two biorders (with $\operatorname{dom}(\tau)=[1, N]$ and $\left.\operatorname{dom}\left(\tau^{\prime}\right)=\left[1, N^{\prime}\right]\right)$. Then $\tau^{\prime}$ is embeddable in $\tau$, if there is a mapping $\varphi: \operatorname{dom}\left(\tau^{\prime}\right) \rightarrow \operatorname{dom}(\tau)$ such that $\varphi$ is an order embedding simultaneously from $\delta_{1}^{\prime}$ into $\delta_{1}$ and from $\delta_{2}^{\prime}$ into $\delta_{2}$.

Example 3.1. Let $\tau=\left(\delta_{1}, \delta_{2}\right)$ be a biorder such that $\delta_{1}=(2,4,1,3)$ and $\delta_{2}=(3,1,2,4)$. Also, let $\tau^{\prime}=\left(\delta_{1}^{\prime}, \delta_{2}^{\prime}\right)$ be a biorder with $\delta_{1}^{\prime}=(3,1,2)$ and $\delta_{2}^{\prime}=(2,1,3)$. Then $\tau^{\prime}$ is embeddable in $\tau$. Indeed, the mapping $\varphi:[1,3] \rightarrow$ $[1,4]$ given by $\varphi(1)=1, \varphi(2)=3$, and $\varphi(3)=4$ is an order embedding from $\delta_{1}^{\prime}$ into $\delta_{1}$ and from $\delta_{2}^{\prime}$ into $\delta_{2}$.

Let $D$ be a set and $m, n \geq 1$ be integers. The set of all $m \times n$ matrices with entries in $D$ is denoted by $D^{m \times n}$. For a matrix $M \in D^{m \times n}$, its entries are denoted by $M_{(i, j)}$ for $i \in[1, m]$ and $j \in[1, n]$. Let $\operatorname{size}(M)=(m, n)$ denote the size of the matrix $M$. Also, let $M_{i}=\left(M_{(i, 1)}, \ldots, M_{(i, n)}\right)$ be the $i$ th row vector and $M_{j}^{T}=\left(M_{(1, j)}, \ldots, M_{(m, j)}\right)$ the $j$ th column vector of $M$. Here the matrix $M^{T}$ is the transpose of $M$ and thus the $j$ th column vector of $M$ equals the $j$ th row vector of $M^{T}$.

A matrix $M \in D^{m \times n}$ is called an $m \times n$-grid, if $D=[1, m n]$ and the entries of $M$ are all distinct, that is, $\left\{M_{(i, j)} \mid i \in[1, m], j \in[1, n]\right\}=[1, m n]$. Hence a grid is a generalization of a permutation to two dimensions.

We shall study biorders $\left(\delta_{1}, \delta_{2}\right)$ that can be represented by grids in such a way that both linear orders can be read from the representing grid. In the following we choose a basic way of reading a grid to produce a biorder. In order not to loose any biorders, such a way of reading must be carefully chosen.

Let $M$ be an $m \times n$-grid. We note first that the row and column vectors $M_{i}$ and $M_{i}^{T}$ can be interpreted as linear orders in a natural way. Then $\left(M_{i}^{T}\right)^{-1}$ is the dual order corresponding to the $i$ th column of $M$, and we 
denote this also by $M_{i}^{-T}$. We define the linear orders $\alpha(M)$ and $\beta(M)$ as follows:

$$
\alpha(M)=\sum_{i=1}^{m} M_{i} \quad \text { and } \quad \beta(M)=\sum_{i=1}^{n} M_{i}^{-T} .
$$

The grid biorder of $M$ is the biorder $\operatorname{Bi}(M)=(\alpha(M), \beta(M))$; see Fig. 1. It is easy to see that, for each row $M_{i}$ and for each column $M_{j}^{T}$ of $M$,

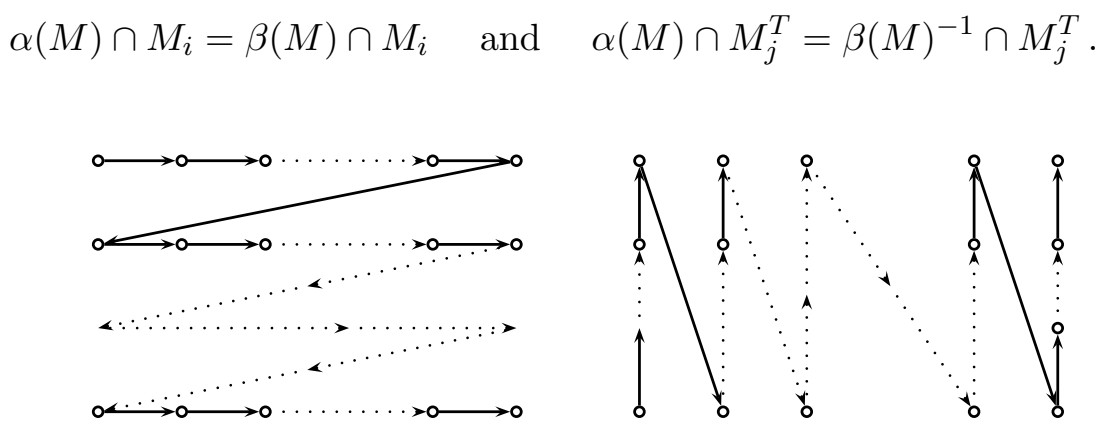

Figure 1: Reading orders of a grid: $\alpha(M)$ and $\beta(M)$.

Example 3.2. (1) Consider the following grid

$$
M=\left(\begin{array}{cccc}
4 & 7 & 2 & 11 \\
5 & 8 & 6 & 9 \\
3 & 10 & 1 & 12
\end{array}\right)
$$

of size $(3,4)$. Then the grid biorder $\operatorname{Bi}(M)=\left(\delta_{1}, \delta_{2}\right)$ of $M$ consists of the following linear orders: $\delta_{1}=(4,7,2,11,5,8,6,9,3,10,1,12)$ and $\delta_{2}=$ $(3,5,4,10,8,7,1,6,2,12,9,11)$.

(2) The grid biorder of a row vector $M=\left(x_{1}, x_{2}, \ldots, x_{n}\right)$ is simply $\operatorname{Bi}(M)=(M, M)$, and for a column vector $M=\left(x_{1}, x_{2}, \ldots, x_{n}\right)^{T}$, we have $\operatorname{Bi}(M)=\left(M, M^{-1}\right)$.

\section{Embedding biorders into grids}

Grids produce rather special biorders $\operatorname{Bi}(M)$ in the sense that the second linear order of $\operatorname{Bi}(M)$ is almost redundant - it is uniquely determined by the first linear order and the size of the grid $M$. Nevertheless, as we shall see, all biorders can be embedded into grid biorders.

Let $\tau=\left(\delta_{1}, \delta_{2}\right)$ be a biorder. We say that a sequence $\left(\sigma_{1}, \sigma_{2}, \ldots, \sigma_{k}\right)$ of partial orders is a left partition of $\tau$ (with $k$ components), if $\delta_{1}=\sum_{i=1}^{k} \sigma_{i}$ and $\sigma_{i} \subseteq \delta_{2}$ for all $i \in[1, k]$. Similarly, a sequence $\left(\kappa_{1}, \kappa_{2}, \ldots, \kappa_{t}\right)$ is a right partition of $\tau$ (with $t$ components), if $\delta_{2}=\sum_{i=1}^{t} \kappa_{i}$ and $\kappa_{i} \subseteq \delta_{1}^{-1}$ for all $i \in[1, t]$. Moreover, a left (right) partition is said to be maximal if it has the smallest number of components among the left (right, respectively) partitions of $\tau$. 
Example 4.1. Let $\tau=\left(\delta_{1}, \delta_{2}\right)$ be the biorder where $\delta_{1}=(1,5,3,4,2)$ and $\delta_{2}=(3,2,1,4,5)$, and set $\sigma_{1}=(1,5), \sigma_{2}=(3,4)$, and $\sigma_{3}=(2)$. Then $\delta_{1}=\sigma_{1} \oplus \sigma_{2} \oplus \sigma_{3}$ and $\sigma_{i} \subseteq \delta_{2}$ for each $i$. Therefore $\left(\sigma_{1}, \sigma_{2}, \sigma_{3}\right)$ is a left partition of $\tau$. Similarly, $\left(\kappa_{1}, \kappa_{2}, \kappa_{3}\right)$ is a right partition of $\tau$, when $\kappa_{1}=$ $(3), \kappa_{2}=(2,1), \kappa_{3}=(4,5)$.

We begin with a lemma concerning pairs of orders contained in grid biorders.

Lemma 4.2. Let $\tau^{\prime}=\operatorname{Bi}(M)$ for an $m \times n$-grid $M$ and let $\tau$ be a biorder such that $\tau=\left(\delta_{1}, \delta_{2}\right) \subseteq \tau^{\prime}$. For each $i \in[1, m]$ and $j \in[1, n]$, let $\sigma_{i}=\delta_{1} \cap M_{i}$ and $\kappa_{j}=\delta_{2} \cap M_{j}^{-T}$. Then $\left(\sigma_{1}, \ldots, \sigma_{m}\right)$ is a left partition and $\left(\kappa_{1}, \ldots, \kappa_{n}\right)$ is a right partition of $\tau$.

Proof. It is clear that $\delta_{1}=\sum_{i=1}^{m} \sigma_{i}$ and $\delta_{2}=\sum_{j=1}^{n} \kappa_{j}$. Since $\tau \subseteq \tau^{\prime}$ and $\sigma_{i} \subseteq M_{i}$, it follows by (1) that $\sigma_{i} \subseteq \delta_{2}$ for each $i$. Similarly, $\kappa_{j} \subseteq \delta_{1}^{-1}$ for each $j$. The claim follows from these observations.

Each biorder $\tau=\left(\delta_{1}, \delta_{2}\right)$ does have a left and a right partition. Indeed, if $\delta_{1}=\left(a_{1}, a_{2}, \ldots, a_{n}\right)$ and $\delta_{2}=\left(b_{1}, b_{2}, \ldots, b_{n}\right)$, then these are trivial left and right partitions: $\delta_{1}=\sum_{i=1}^{n}\left(a_{i}\right)$ and $\delta_{2}=\sum_{i=1}^{n}\left(b_{j}\right)$ where each $\left(a_{i}\right)$ and $\left(b_{j}\right)$ is a one element linear order. We shall prove in the following that the maximal left and right partitions of a biorder $\tau$ are unique.

Lemma 4.3. Let $\tau$ be a biorder and let $\left(\sigma_{1}, \ldots, \sigma_{k}\right)$ and $\left(\kappa_{1}, \ldots, \kappa_{t}\right)$ be a left and a right partition of $\tau$, respectively. Then $\operatorname{dom}\left(\sigma_{i} \cap \kappa_{j}\right)$ has at most one element for each $i \in[1, k]$ and $j \in[1, t]$.

Proof. Let $\tau=\left(\delta_{1}, \delta_{2}\right)$. Suppose that there are elements $a, b \in \operatorname{dom}(\tau)$ such that $(a, b) \in \sigma_{i}$ and $\{a, b\} \subseteq \operatorname{dom}\left(\kappa_{j}\right)$ for some $i \in[1, k]$ and $j \in[1, t]$. Since $\sigma_{i} \subseteq \delta_{1}$, also $(a, b) \in \delta_{1}$. On the other hand, $\sigma_{i} \subseteq \delta_{2}$ and $\kappa_{j} \subseteq \delta_{2}$, and therefore $(a, b) \in \kappa_{j}$. However, by definition, $\kappa_{j} \subseteq \delta_{1}^{-1}$ and thus $(b, a) \in \delta_{1}$, which implies that $a=b$ proving the claim.

Let $\sigma=\left(\sigma_{1}, \ldots, \sigma_{k}\right)$ and $\kappa=\left(\kappa_{1}, \ldots, \kappa_{t}\right)$ be left and right partitions of a biorder $\tau$. If $\operatorname{dom}\left(\sigma_{i} \cap \kappa_{j}\right) \neq \emptyset$, then, by Lemma 4.3 , the intersection has exactly one element. In this case, we say that the pair $(i, j)$ is compatible in $(\sigma, \kappa)$. Trivially, every element $x \in \operatorname{dom}(\tau)$ belongs to exactly one set $\operatorname{dom}\left(\sigma_{i} \cap \kappa_{j}\right)$. Therefore we can define $\chi_{\tau}: \operatorname{dom}(\tau) \rightarrow[1, k] \times[1, t]$ by

$$
\chi_{\tau}(x)=(i, j) \text { if } x=\sigma_{i} \cap \kappa_{j} .
$$

We now give necessary and sufficient conditions for a biorder $\tau$ to be embeddable into a given grid biorder.

Theorem 4.4. Let $\tau=\left(\delta_{1}, \delta_{2}\right)$ be a biorder and let $\tau^{\prime}=\operatorname{Bi}(M)$ be a grid biorder for an $m \times n$-grid $M$. The following two conditions are equivalent for each function $\varphi: \operatorname{dom}(\tau) \rightarrow \operatorname{dom}\left(\tau^{\prime}\right)$.

(i) $\varphi$ is an embedding of $\tau$ into $\tau^{\prime}$. 
(ii) There are integers $k$ and $t$, and left and right partitions $\sigma=\left(\sigma_{1}, \ldots, \sigma_{k}\right)$ and $\kappa=\left(\kappa_{1}, \ldots, \kappa_{t}\right)$ of $\tau$, and order embeddings $\pi_{1}:[1, k] \rightarrow[1, m]$ and $\pi_{2}:[1, t] \rightarrow[1, n]$ such that for all compatible pairs $(i, j)$ of $(\sigma, \kappa)$,

$$
\varphi\left(\sigma_{i} \cap \kappa_{j}\right)=M_{\left(\pi_{1}(i), \pi_{2}(j)\right)} .
$$

Proof. Let $\tau^{\prime}=\left(\delta_{1}^{\prime}, \delta_{2}^{\prime}\right)$.

(1) Assume first that $\varphi$ is an embedding. Let, for each $i \in[1, m]$ and $j \in[1, n]$,

$$
\sigma_{i}^{\prime}=\varphi\left(\delta_{1}\right) \cap M_{i} \text { and } \kappa_{j}^{\prime}=\varphi\left(\delta_{2}\right) \cap M_{j}^{-T} .
$$

Moreover, let $d_{1}<d_{2}<\cdots<d_{k}$ be the increasing sequence of all indices such that $\sigma_{d_{1}}^{\prime}, \ldots, \sigma_{d_{k}}^{\prime} \neq \emptyset$, and let $e_{1}<e_{2}<\cdots<e_{t}$ be the increasing sequence of all indices such that $\kappa_{e_{1}}^{\prime}, \ldots, \kappa_{e_{t}}^{\prime} \neq \emptyset$. By Lemma 4.2, $\sigma=$ $\left(\sigma_{d_{1}}, \ldots, \sigma_{d_{k}}\right)$ is a left partition and $\kappa=\left(\kappa_{e_{1}}, \ldots, \kappa_{e_{t}}\right)$ is a right partition of $\varphi(\tau)$. It is immediate that $M_{\left(d_{i}, e_{j}\right)}=\operatorname{dom}\left(\sigma_{d_{i}}^{\prime} \cap \kappa_{e_{j}}^{\prime}\right)$ for all compatible pairs $\left(d_{i}, e_{j}\right)$ (where $i \in[1, k]$ and $j \in[1, t]$ ).

Let $\pi_{1}:[1, k] \rightarrow[1, m]$ and $\pi_{2}:[1, t] \rightarrow[1, n]$ be defined by $\pi_{1}(i)=d_{i}$ and $\pi_{2}(j)=e_{j}$, respectively. Obviously, $\pi_{1}$ and $\pi_{2}$ are order embeddings. Let $\sigma_{i}=\varphi^{-1}\left(\sigma_{d_{i}}^{\prime}\right)$ and $\kappa_{j}=\varphi^{-1}\left(\kappa_{e_{j}}^{\prime}\right)$ for $i \in[1, k]$ and $j \in[1, t]$. Because $\varphi$ is an embedding of $\tau$ onto $\varphi(\tau),\left(\sigma_{1}, \ldots, \sigma_{k}\right)$ is a left partition and $\left(\kappa_{1}, \ldots, \kappa_{t}\right)$ is a right partition of $\tau$. Now, if $x \in \operatorname{dom}\left(\sigma_{i} \cap \kappa_{j}\right)$, then we have $\varphi(x) \in$ $\operatorname{dom}\left(\sigma_{\pi_{1}(i)}^{\prime} \cap \kappa_{\pi_{2}(j)}^{\prime}\right)$. Hence, by the above, $\varphi(x)=M_{\left(\pi_{1}(i), \pi_{2}(j)\right)}$ for each compatible pair $(i, j)$ with $i \in[1, k]$ and $j \in[1, t]$ as required.

(2) Suppose now that (ii) is satisfied. (Note that $\varphi$ is well defined by Lemma 4.3.) The injectivity of $\varphi$ follows directly from its definition and from Lemma 4.3. We need only to show that $\varphi$ is order preserving for both linear orders of $\tau$.

Let $x, y \in \operatorname{dom}(\tau)$, and let $i, p \in[1, k]$ and $j, q \in[1, t]$ be such that $x=\operatorname{dom}\left(\sigma_{i} \cap \kappa_{j}\right)$ and $y=\operatorname{dom}\left(\sigma_{p} \cap \kappa_{q}\right)$. If $(x, y) \in \delta_{1}$ then $i \leq p$, and therefore $\pi_{1}(i) \leq \pi_{1}(p)$, since $\pi_{1}$ is order preserving. Moreover, if $i=p$, then also $(x, y) \in \delta_{2}$, since $\sigma_{i} \subseteq \delta_{2}$. Hence in this case, $q \leq j$ and also $\pi_{2}(q) \leq \pi_{2}(j)$, since $\pi_{2}$ is order preserving. It follows then that

$$
\varphi(x, y)=(\varphi(x), \varphi(y))=\left(M_{\left(\pi_{1}(i), \pi_{2}(j)\right)}, M_{\left(\pi_{1}(p), \pi_{2}(q)\right)}\right) \in \delta_{1}^{\prime} .
$$

Similarly, if $(x, y) \in \delta_{2}$, then $j \leq q$ and thus $\pi_{2}(j) \leq \pi_{2}(q)$. Moreover, if $q=j$, then $(x, y) \in \delta_{1}^{-1}$, since $\kappa_{j} \subseteq \delta_{1}^{-1}$. In this case, $i \leq p$ and also $\pi_{1}(i) \leq \pi_{1}(p)$. As in the above we have now that $\varphi(x, y) \in \delta_{2}^{\prime}$. We conclude that $\varphi$ is an embedding from $\tau$ into $\tau^{\prime}$.

We note that, in the notations of the previous theorem, the grid biorder $\operatorname{Bi}(M)$ into which the given biorder $\tau$ is embeddable, has the size at least $(k, t)$ where $k$ and $t$ are the numbers of the components of the left and right partitions, respectively. 
Example 4.5. Consider the grid

$$
M=\left(\begin{array}{lll}
3 & 5 & 1 \\
4 & 2 & 6
\end{array}\right)
$$

for which we have $\operatorname{Bi}(M)=((3,5,1,4,2,6),(4,3,2,5,6,1))$. Let also $\tau=$ $((3,2,1),(3,1,2))$. Then $\tau$ has a left partition $((3,2),(1))$ and a right partition $((3),(1),(2))$. Now $\tau$ can be embedded into $\operatorname{Bi}(M)$ by the embedding $\varphi$ defined by $\varphi(1)=2, \varphi(2)=1$ and $\varphi(3)=3$. Indeed, $\varphi(3,2,1)=(3,1,2)$ is a suborder of $(3,5,1,4,2,6)$ and $\varphi(3,1,2)=(3,2,1)$ is a suborder of $(4,3,2,5,6,1)$. In this case, the order embeddings $\pi_{1}:[1,2] \rightarrow[1,2]$ and $\pi_{2}:[1,3] \rightarrow[1,3]$ of Theorem 4.4 are both identity functions. For instance, we have that $\sigma_{1}=(3,2)$ and $\kappa_{3}=(2)$, and therefore

$$
M_{(1,3)}=1=\varphi(2)=\varphi\left(\sigma_{1} \cap \kappa_{3}\right)=M_{\left(\pi_{1}(1), \pi_{2}(3)\right)},
$$

and hence $\pi_{1}(1)=1$ and $\pi_{2}(3)=3$.

The grid biorder $\operatorname{Bi}(M)$ is not the smallest one into which $\tau$ can be embedded. It is easy to verify that $\tau$ can be embedded into $\operatorname{Bi}\left(M^{\prime}\right)$ where the grid $M^{\prime}$ has size $(2,2)$ :

$$
M^{\prime}=\left(\begin{array}{ll}
3 & 2 \\
4 & 1
\end{array}\right)
$$

Here $\operatorname{Bi}\left(M^{\prime}\right)=((3,2,4,1),(4,3,1,2))$. The embedding $\varphi^{\prime}$ is the identity function in this case.

The following result proves that every left (right) partition of a biorder $\tau$ can be extended to a unique maximum left (right, respectively) partition.

Lemma 4.6. Each biorder $\tau$ possesses a unique maximal left partition and a unique maximal right partition.

Proof. Let $\tau=\left(\delta_{1}, \delta_{2}\right)$. We prove the claim for left partitions; for right partitions the proof is similar and omitted here. Now there exists at least one left partition for $\tau$; namely the trivial left partition. Let then $\left(\sigma_{1}, \ldots, \sigma_{k}\right)$ be any left partition of $\tau$. If for some $i \in[1, k-1], \sigma_{i} \oplus \sigma_{i+1} \subseteq \delta_{2}$, then $\sigma_{i} \oplus \sigma_{i+1} \subseteq \delta_{1}$, and hence also $\left(\sigma_{1}, \ldots,\left(\sigma_{i} \oplus \sigma_{i+1}\right), \ldots, \sigma_{k}\right)$ is a left partition of $\tau$, and it has $k-1$ components. We may thus assume that in the chosen left partition there are no indices $i \in[1, k-1]$ such that $\sigma_{i} \oplus \sigma_{i+1} \subseteq \delta_{2}$. Let $\left(\sigma_{1}^{\prime}, \ldots, \sigma_{p}^{\prime}\right)$ be another left partition of $\tau$. If for some $i \in[1, p]$ and $j \in[1, k-1], \operatorname{dom}\left(\sigma_{i}^{\prime}\right) \cap \operatorname{dom}\left(\sigma_{j}\right) \neq \emptyset$ and $\operatorname{dom}\left(\sigma_{i}^{\prime}\right) \cap \operatorname{dom}\left(\sigma_{j+1}\right) \neq \emptyset$, then $(a, b) \in \sigma_{i}^{\prime}$ for the maximal element $a$ of $\sigma_{j}$ and the minimal element $b$ of $\sigma_{j+1}$. Since $\sigma_{j} \subseteq \delta_{2}, \sigma_{j+1} \subseteq \delta_{2}$ and $(a, b) \in \delta_{2}$, evidently also $\sigma_{j} \oplus \sigma_{j+1} \subseteq \delta_{2}$, contradicting our assumption. Consequently, for each $i \in[1, p]$, we have $\sigma_{i}^{\prime} \subseteq \sigma_{j}$ for some $j \in[1, k]$. Thus $\left(\sigma_{1}, \ldots, \sigma_{k}\right)$ is a maximal left partition and it is unique as such a partition.

Let $\left(\sigma_{1}, \ldots, \sigma_{k}\right)$ and $\left(\kappa_{1}, \ldots, \kappa_{t}\right)$ be the maximal left and right partitions of a biorder $\tau$, respectively. Then $\operatorname{size}^{P}(\tau)=(k, t)$ is called the partitive size of $\tau$. By Lemma 4.6, this notion is well defined for each biorder $\tau$.

From Theorem 4.4 we deduce the following estimation on the size for the grid biorders into which a given biorder $\tau$ can be embedded. 
Lemma 4.7. Let $\tau$ be a biorder $\tau$ that is embeddable into a grid biorder $\operatorname{Bi}(M)$ for a grid $M$. Then $\operatorname{size}^{P}(\tau) \leq \operatorname{size}(M)$.

Proof. The existence of the injective functions $\pi_{1}$ and $\pi_{2}$ in Theorem 4.4 implies that $\operatorname{size}(M) \geq(k, t)$ where $k$ and $t$ equal the number of components in the left and right partitions given by Theorem 4.4. Since the maximal left and right partitions of $\tau$ have the least number of components, we have $\operatorname{size}(M) \geq(k, t) \geq \operatorname{size}^{P}(\tau)$.

We are going to show now that each biorder can be embedded into a unique grid biorder 'modulo $\tau$ '. First we define the meaning of 'modulo $\tau$ '.

Let $\tau$ be a biorder on $[1, N]$, and let $M^{\prime}$ and $M^{\prime \prime}$ be two $m \times n$-grids. Then $\operatorname{Bi}\left(M^{\prime}\right)$ and $\operatorname{Bi}\left(M^{\prime \prime}\right)$ are said to be congruent modulo $\tau$, if $\tau \subseteq \operatorname{Bi}\left(M^{\prime}\right)$ and $\tau \subseteq \operatorname{Bi}\left(M^{\prime \prime}\right)$, and the elements of $[1, N]$ are in the same places in the grids $M^{\prime}$ and $M^{\prime \prime}$, i.e., if $M_{(i, j)}^{\prime}=M_{(i, j)}^{\prime \prime}$ for each $M_{(i, j)}^{\prime} \in[1, N]$ with $(i, j) \in$ $[1, m] \times[1, n]$.

Example 4.8. Let $\tau^{\prime}=\operatorname{Bi}\left(M^{\prime}\right)$ and $\tau^{\prime \prime}=\operatorname{Bi}\left(M^{\prime \prime}\right)$ be grid biorders where

$$
M^{\prime}=\left(\begin{array}{lll}
1 & 2 & 5 \\
4 & 3 & 6
\end{array}\right) \quad \text { and } \quad M^{\prime \prime}=\left(\begin{array}{lll}
5 & 1 & 2 \\
4 & 6 & 3
\end{array}\right) .
$$

For the biorder $\tau=((1,2,3),(1,3,2))$, we have $\tau \subseteq \tau^{\prime}$ and $\tau \subseteq \tau^{\prime \prime}$. However, $\tau^{\prime}$ and $\tau^{\prime \prime}$ are not congruent modulo $\tau$, because $M_{(1,1)}^{\prime}=1 \in[1,3]$, but $M_{(1,1)}^{\prime} \neq M_{(1,1)}^{\prime \prime}$. (Indeed, we have even that $M_{(1,1)}^{\prime \prime} \notin[1,3]$.)

We are now ready to express our main embedding theorem which states that every biorder can be embedded into a unique smallest grid biorder where uniqueness is taken up to congruence of biorders.

Theorem 4.9. Let $\tau$ be a biorder on $D=[1, N]$ with $\operatorname{size}^{P}(\tau)=(k, t)$. There exists a $k \times t$-grid $M^{\prime}$ such that

(i) $\tau \subseteq \operatorname{Bi}\left(M^{\prime}\right)$, and

(ii) if $\tau \subseteq \operatorname{Bi}\left(M^{\prime \prime}\right)$ for a grid $M^{\prime \prime}$, then either $\operatorname{Bi}\left(M^{\prime \prime}\right)$ and $\operatorname{Bi}\left(M^{\prime}\right)$ are equivalent modulo $\tau$ or $\operatorname{size}\left(M^{\prime \prime}\right)>\operatorname{size}^{P}(\tau)$.

Proof. Let $\tau=\left(\delta_{1}, \delta_{2}\right)$ and let $\left(\sigma_{1}, \ldots, \sigma_{k}\right)$ and $\left(\kappa_{1}, \ldots, \kappa_{t}\right)$ be the maximal left and right partitions of $\tau$, respectively. Moreover, we let $D^{\prime}=[N+1, k t]$. For each pair $(i, j) \in[1, k] \times[1, t]$, let $\mu(i, j)$ be the number of pairs $(r, s)$ such that $(r, s) \leq(i, j)$ and $\operatorname{dom}\left(\sigma_{r} \cap \kappa_{s}\right)=\emptyset$, i.e., for which $\chi_{\tau}^{-1}(r, s)$ is not defined. By Lemma 4.3, $\chi_{\tau}^{-1}(r, s)$ is undefined for exactly $\left|D^{\prime}\right|=k t-N$ pairs $(r, s)$.

Define a $k \times t$-grid $M^{\prime}$ as follows: for each pair $(i, j) \in[1, k] \times[1, t]$,

$$
M_{(i, j)}^{\prime}= \begin{cases}\chi_{\tau}^{-1}(i, j), & \text { if } \sigma_{i} \cap \kappa_{j} \neq \emptyset, \\ N+\mu(i, j), & \text { if } \sigma_{i} \cap \kappa_{j}=\emptyset .\end{cases}
$$

Denote $\tau^{\prime}=\operatorname{Bi}\left(M^{\prime}\right)$. 
We apply Theorem 4.4 for the case where $\pi_{1}(i)=i$ and $\pi_{2}(i)=i$ for each $i$, and accordingly we define $\varphi(x)=M_{\left(\pi_{1}(i), \pi_{2}(j)\right)}^{\prime}$ where $(i, j)=\chi_{\tau}(x)$. By Theorem 4.4, $\varphi$ is an embedding of $\tau$ into $\tau^{\prime}$. Now, $\varphi$ is the identity function on $\operatorname{dom}(\tau)$, and thus $\tau \subseteq \tau^{\prime}$.

Suppose then that $\tau$ is contained in another grid biorder $\tau^{\prime \prime}=\operatorname{Bi}\left(M^{\prime \prime}\right)$ for a $p \times q$-grid $M^{\prime \prime}$. By Lemma 4.7 , we have that $(k, t) \leq(p, q)$.

Suppose now that $(k, t)=(p, q)$. By applying Theorem 4.4 to the identity function $\varphi$ as an embedding of $\tau$ into $\tau^{\prime \prime}$, we have that the left and right partitions in this case are necessarily the maximal left and right partitions of $\tau$, because there are exactly $k$ and $t$ components, respectively. Moreover, necessarily $\pi_{1}(i)=i$ and $\pi_{2}(i)=i$ for all $i$, that is, $M_{(i, j)}^{\prime \prime}=\chi_{\tau}^{-1}(i, j)$ for each compatible pair $(i, j)$. Hence $M_{(i, j)}^{\prime}=M_{(i, j)}^{\prime \prime}$ for all compatible pairs $(i, j)$, which proves the claim.

The next example illustrates the construction given in Theorem 4.9.

Example 4.10. Let $\tau=\left(\delta_{1}, \delta_{2}\right)$ be a biorder with $\delta_{1}=(3,5,1,6,2,4)$ and $\delta_{2}=(2,1,3,4,6,5)$. Then the maximal left and right partitions of $\tau$ are $\left(\sigma_{1}, \sigma_{2}, \sigma_{3}\right)$ and $\left(\kappa_{1}, \kappa_{2}\right)$, where $\sigma_{1}=(3,5), \sigma_{2}=(1,6), \sigma_{3}=(2,4)$, and $\kappa_{1}=(2,1,3), \kappa_{2}=(4,6,5)$. Hence $\operatorname{size}^{P}(\tau)=(3,2)$ and the entries $M_{(i, j)}=\operatorname{dom}\left(\sigma_{i} \cap \kappa_{j}\right)$ in the grid obtained in the proof of Theorem 4.9 are: $M_{(1,1)}=3, M_{(1,2)}=5, M_{(2,1)}=1, M_{(2,2)}=6, M_{(3,1)}=2, M_{(3,2)}=4$. Hence $\tau=\operatorname{Bi}(M)$ for the grid

$$
M=\left(\begin{array}{ll}
3 & 5 \\
1 & 6 \\
2 & 4
\end{array}\right) .
$$

The previous result states that the size of the smallest grid biorder, which contains a given biorder $\tau$, is unique, and the grid biorder itself is 'unique modulo $\tau$ '. We shall now 'forget' the elements from the grids that will not be in the domain of $\tau$ by introducing a special free symbol $\star$. Let $S$ be any set excluding the free symbol $\star$, and let $A \subseteq S$. Define a general purpose function $\Lambda_{A}: S \rightarrow S \cup\{\star\}$ by

$$
\Lambda_{A}(s)= \begin{cases}s & \text { if } s \in A, \\ \star & \text { if } a \notin A .\end{cases}
$$

Given a biorder $\tau=\left(\delta_{1}, \delta_{2}\right)$ and a subset $A \subseteq \operatorname{dom}(\tau)$, a pair $\Lambda_{A}(\tau)=$ $\left(\Lambda_{A}\left(\delta_{1}\right), \Lambda_{A}\left(\delta_{2}\right)\right)$ is called a biorder with free symbols obtained from $\tau$ by $A$. Similarly, for a grid $M$ the matrix $\Lambda_{A}(M)$ is a matrix with free symbols.

Example 4.11. Let $\tau=((1,2,3,4),(2,4,1,3))$ be a biorder and choose $A=\{1,4\}$. Then $\Lambda_{A}(\tau)=((1, \star, \star, 4),(\star, 4,1, \star))$. The biorder $\tau$ is a grid biorder and the corresponding grid $M$ together with the matrix $\Lambda_{A}(M)$ are given below:

$$
M=\left(\begin{array}{ll}
1 & 2 \\
3 & 4
\end{array}\right) \quad \text { and } \quad \Lambda_{A}(M)=\left(\begin{array}{cc}
1 & \star \\
\star & 4
\end{array}\right)
$$


Using the notation of a free symbol, if two grid biorders $\tau^{\prime}$ and $\tau^{\prime \prime}$ are congruent modulo a biorder $\tau$ with a domain $D$, then $\Lambda_{D}\left(\tau^{\prime}\right)=\Lambda_{D}\left(\tau^{\prime \prime}\right)$.

Let $\tau \subseteq \tau^{\prime}$ for a biorder $\tau^{\prime}$ and let $\operatorname{dom}(\tau)=D$. The biorder $\Lambda_{D}\left(\tau^{\prime}\right)$ with free symbols is called a cover of $\tau$. Furthermore, if $\tau^{\prime}$ is a grid biorder, then $\Lambda_{D}\left(\tau^{\prime}\right)$ is said to be a matrix cover of $\tau$. A matrix cover of $\tau$ is a minimal matrix cover, if $\tau^{\prime}$ has minimal size.

Theorem 4.9 can now be restated as follows.

Theorem 4.12. For each biorder there exists a unique minimal matrix cover.

Proof. The claim is obvious by Theorem 4.9 and the above definitions.

Example 4.13. Let $\tau=\left(\delta_{1}, \delta_{2}\right)$ be a biorder with $\delta_{1}=(4,5,3,1,2,6)$ and $\delta_{2}=(2,3,1,6,4,5)$. Then the maximal left and right partitions of $\tau$ are $\left(\sigma_{1}, \sigma_{2}, \sigma_{3}\right)$ and $\left(\kappa_{1}, \kappa_{2}, \kappa_{3}, \kappa_{4}\right)$, where $\sigma_{1}=(4,5), \sigma_{2}=(3,1), \sigma_{3}=(2,6)$, and $\kappa_{1}=(2,3), \kappa_{2}=(1), \kappa_{3}=(6,4), \kappa_{4}=(5)$. Now $\operatorname{size}^{P}(\tau)=(3,4)$ and the entries in the matrix given by Theorem 4.9 are $M_{(1,3)}=4, M_{(1,4)}=5$, $M_{(2,1)}=3, M_{(2,2)}=1, M_{(3,1)}=2, M_{(3,3)}=6$. The rest of the entries are filled with the free symbol $\star$. Hence the minimal matrix cover of $\tau$ is the grid biorder $\operatorname{Bi}(M)$ for the grid

$$
M=\left(\begin{array}{cccc}
\star & \star & 4 & 5 \\
3 & 1 & \star & \star \\
2 & \star & 6 & \star
\end{array}\right) .
$$

Acknowledgements. T. Harju gratefully acknowledges the support of the Academy of Finland under project 39802. G. Rozenberg gratefully acknowledges partial support by NSF grant 0121422 .

\section{References}

[1] A. Ehrenfeucht, T. Harju and G. Rozenberg, The Theory of 2-Structures, World Scientific, 1999.

[2] A. Ehrenfeucht, T. Harju, P. ten Pas and G. Rozenberg, Permutations, parenthesis words, and Schröder numbers, Discrete Math. 190 (1998), $259-264$.

[3] A. Ehrenfeucht and G. Rozenberg, T-structures, T-functions, and texts, Theoret. Comput. Sci. 116 (1993), 227 - 290.

[4] A. Pnueli, A. Lempel and S. Even, Transitive orientation of graphs and identification of permutation graphs, Canad. J. Math. 23 (1971), 160 175 .

[5] W. T. Trotter, Combinatorics and Partially Ordered Sets. Dimension Theory, The Johns Hopkins Univ. Press, Baltimore, 1992. 


\section{TURKU}

\section{CENTRE for}

COMPUTER

SCIENCE

Lemminkäisenkatu 14 A, 20520 Turku, Finland | www.tucs.fi

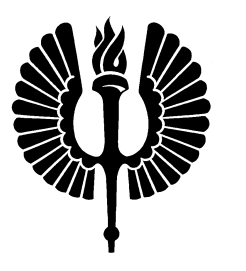

University of Turku

- Department of Information Technology

- Department of Mathematical Sciences

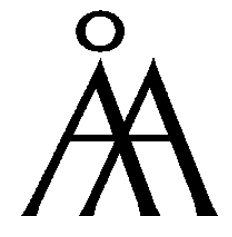

Åbo Akademi University

- Department of Computer Science

- Institute for Advanced Management Systems Research

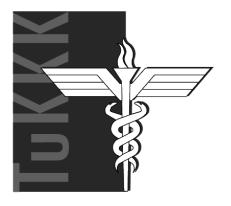

Turku School of Economics and Business Administration

- Institute of Information Systems Sciences

ISBN 952-12-1452-X

ISSN 1239-1891 